\title{
PRECURSORES DE LA ENSEÑANZA DEL URBANISMO EN CHILE
} PERIODO 1928 - 1953

\author{
María Isabel Pavez R.
}

\begin{abstract}
Indagando en algunas de las circunstancias que dieron origen a la enseñanza del Urbanismo en la Escuela de Arquitectura de la Universidad de Chile en los años veinte y, posteriormente, a su institucionalización en los años cincuenta, se evoca el rol que cuatro destacados arquitectos cumplieron para ello. Tanto por su acción individual, como por su acción conjunta en virtud de los lazos intelectuales y profesionales que los unieron en diversos momentos, hicieron valiosos aportes al medio académico y profesional.
\end{abstract}

Advenimiento del campo temático del Urbanismo en la "Escuela de Arquitectura de la Facultad de Ciencias Fisicas y Matemáticas" de la Universidad de Chile en la década de los años veinte.

aprimera guerra mundial,yelestablecimiento de las economías de guerra, suscitaron reflexiones sobre la necesidad de planificar el desarrollo económico y social de las naciones. La gran crisis mundial de los años treinta, fué un acontecimiento decisivo para los diversos Estados al respecto. El nacimiento de la economía política, la emergencia de los debates sobre el dirigismo y sobre el espacio social productivo del Estado, las polémicas sobre el liberalismo y el estatismo y tantos otros temas, estuvieron presentes en la definición de nuevas formas de vida y en los planteamientos de nuevas teorías y opciones urbanísticas y habitacionales.

Los tratados teóricos sobre Urbanismo sobrepasaron entonces el interés en la tradición histórica y en sus valores estéticos, para avanzar rápidamente a una concepción socioeconómica, técnica y política de lo que es la vida urbana y sus fenómenos. La segunda guerra mundial, con su enorme destrucción y consecuente reconstrucción masiva provocó una preocupación aún mayor por las tareas de Planificación, desarrollándoseenlospaísesmás afectados centros de investigación urbanística y habitacional con el fin de orientar la reconstrucción de sus ciudades y aldeas, además de numerosas instituciones destinadas a llevar a cabo dicha reconstrucción.
Paralelamente, en Chile se agudizaban los fenómenos de crecimiento urbano causados indirectamente por aquellos mismos hechos históricos. Por su gravedad, la crisis de los años treinta representó la línea demarcatoria que vino a indicar el agotamiento del modelo de crecimiento exportador de materias primas, y el tránsito hacia el denominado "modelo de industrialización por sustitución de importaciones". El impacto que provocó sobre nuestra red urbana el traspaso de una economía rural a otra industrial, tipicamente urbana, entre otros fenómenos en el caso chileno, rompió definitivamente el relativo equilibrio del sistemaurbano de fines del siglo pasado, volcando al país en una acelerada urbanización, marcada por una creciente concentración de población y de actividades en ciertas ciudades, hoy metrópolis.

Al comenzar a manifestarse este fenómeno, el país, al igual que el resto del mundo, y aún los países más desarrollados, no tenía doctrinas orientadoras ni herramientas para actuar, no se conocian en la época los Planos Reguladores o Directores propiamente tales, por falta de legislación apropiada, de enseñanza sistemática y de experiencia científica, ni siquiera había conciencia generalizada de la gravedad del proceso que se estaba desarrollando en nuestro territorio. Sin embargo, las Escuelas de Arquitectura del país vislumbraron rápidamente la nueva realidad que se configuraba y comprendieron pronto el rol que les correspondería llevar a cabo desde el ámbito universitario. Asi, en 1928, ya se había creado el primer CURSO DE URBANISMO en la Escuela de Arquitectura de la Universidad de Chile, por iniciativa del Arquitecto Alberto Schade Pohlenz, quien formuló un programa inspirado en sus conocimientos de urbanística, y, en parte. en los planteamientos del urbanista vienés Camilo Sitte (1843 - 1903). Ello ocurría varios lustros antes incluso, de la existencia de cursos similares en otras universidades americanas y aún europeas.

Por otra parte, y como consecuencia del terremoto de Talca, Constitución y Curicó en 1928, se dictó la primera LEY GENERAL DE CONSTRUCCIONES Y URBANIZACION № 4.563, del 30 de Enero de 1929, promovida por el Ministro de Fomento de la época, Don Luis Schmidt Quezada, siendo su principal redactor el Arquitecto Luis Muñoz Maluschka. (Presidencia de Ibáñez). El Ministro Schmidt comprendió el grave problema de la anarquía técnica y administrativa que existía en el control de las actividades de la edificación parti- cular. La Ley citada estableció, por primera vez en Chile y en Sudamérica, la obligación general a todas las Comunas con poblaciones sobre veinte mil habitantes, de confeccionar un plano de urbanización, que debía ser sometido a la aprobación del Ejecutivo. La Ordenanza General de esta Ley estableció las características que debían tener estos planos, y las condiciones a que se deberían ceñir los trazados de las nuevas poblaciones. Las conquistas del Urbanismo internacional se vieron reflejadas en el capítulo VIII de la Ordenanza General, desarrollado con la colaboración del Director del Departamento de Arquitectura, Arquitecto Hermógenes del Canto. Se establecía allí la necesidad de formar barrios residenciales, comerciales, obrerose industriales, como base del agrado de vida en una ciudad perfeccionada.

Influencia decisiva en la evolución de las acciones iniciadas por los profesionales chilenos en beneficio de la urbanización de nuestras ciudades, tuvo la llegada al país del destacado Arquitecto, Ingeniero Civil y Urbanista vienés Dr. Karl H. Brunner en elaño 1929. A partir de la iniciativa del Arquitecto Rodulfo Oyarzún Philippi, ex-alumno de Brunner en Viena, Muñoz Maluschka intervino en la Dirección de Obras Públicas, ante el Ministro Schmidt Quezada, quien también era Profesor de la Escuela de Arquitectura-, para efectos de la contratación de Brunner como Consejero Urbanista del Gobierno y también como Profesor para la Universidad de Chile.

El Urbanismo científico funcionalista era en general desconocido en Chile antes de la llegada de Brunner, considerándose hasta entonces que la acción urbanística debía encaminarse sólo hacia el ornato y el embellecimiento de las ciudades. El Urbanismo como ciencia de las correlaciones técnicas y administrativas cuya finalidad es el agrado y eficiencia de la vida urbana, no se había desarrollado aún en nuestro medio.

El Dr. Brunner, quien era una autoridad en materia de Urbanismo, planteó los problemas urbanos de Santiago y programó los estudios necesarios para abordar su resolución, entregando al Gobierno, sobre trescientos informes. donde eran tratados los problemas de las áreas verdes, de la extensión y crecimiento urbanos, del ferrocarril de circunvalación, de las zonas industriales, del futuro Metropolitano, y de un sinnúmero de problemas, muchos de los cuales todavía hoy, después de sesenta años. aguardan la oportunidad de una solución satisfactoria. 


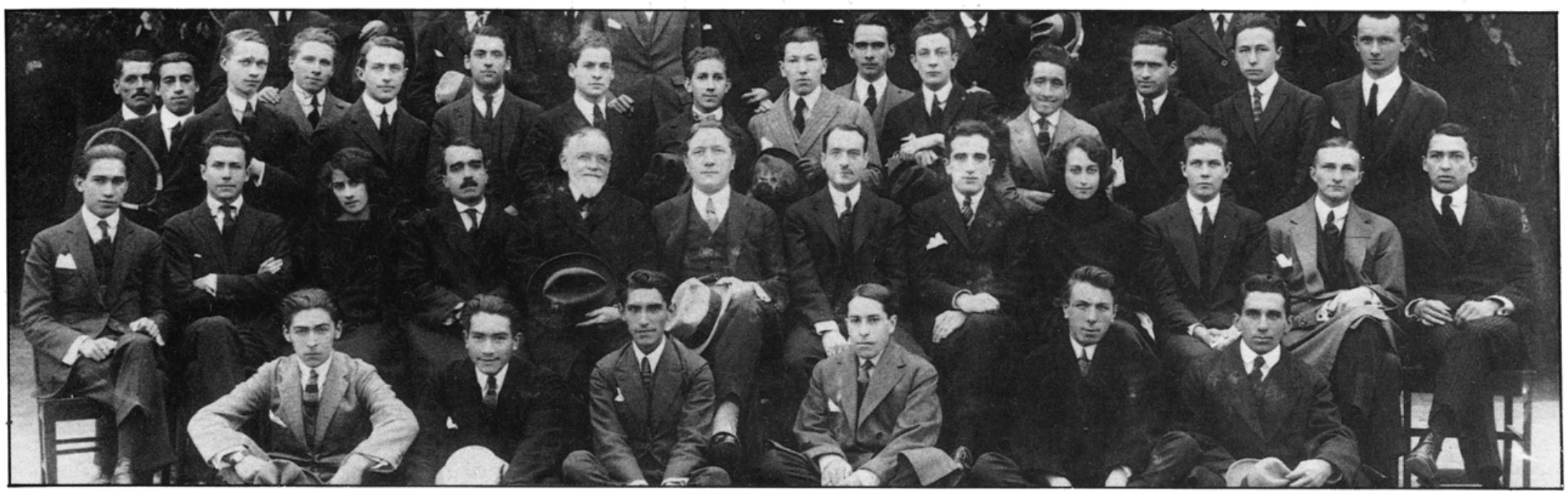

Además de lo señalado, en su primera y corta estada en Chile, el Dr. Brunner estudió los Planos Reguladores de Providencia, San Miguel, Valdivia y Talcahuano. Abordó también grandes proyectos para Santiago, como el Barrio Cívico, en 1930, que sería elaborado luego en forma definitiva por el Arqto.. Carlos Vera dando una mayor altura al conjunto; la Diagonal Oriente; el traslado de la Estación Ferroviaria Pirque, para crear una de las primeras acciones de remodelación con el Parque Bustamante; y el aislamiento del Cerro Santa Lucía, entre otros.

En cuanto a su proyecto de Plano Regulador de la Ciudad de Santiago, desarrollado en su segunda estada en Chile, es destacable su proposición con un criterio de mayor alcance el de un Plan Metropolitano. Brunner intenvino también, y de manera directa, en la formula ción de la nueva LEY GENERAL DE CONSTRUCCIONES Y URBANIZACION Y SU ORDENANZA GENERAL, D.F.L. №345 de 30 de Mayo de 1931. En la nueva Ley se perfeccionó y se le dió base legal a numerosas facultades que la Ley $\mathrm{N}$ 4.563, no le confirmó a los Municipios, para poder establecer un control eficiente sobre la edificación particular.

Su labor en el ámbito universitario tuvo por resultado la creación del PRIMER SEMINARIO DE URBANISMO realizado en Chile y América Latina. El Dr. Brunner llevó a cabo este curso intensivo de preparación de la primera generación de Urbanistas que tuno el país a nivel de graduados, los que dieron con sus realizaciones profesionales, un significativo impulso al desarrollo de la cultura y técnica del urbanismo nacional. A la partida de Brunner, Oyarzún Philippi, uno de sus colaboradores, asumió la Cátedra de Urbanismo con gran brillo entre 1932 y 1946.

A estas actividades predecesoras, se sumaron las orientaciones doctrinarias de los Congresos Internacionales de Arquitectura Moderna, iniciados en 1928. En el medio nacional, el INSTITUTO NACIONAL DE URBANISMO, creado en 1929 y donde participaron activamente nuestros profesores precursores de la enseñanza del urbanismo en Chile, llevó la vanguardia de la preocupación nacional por el Urbanismo. La reforma de la enseñanza de la Arquitectura en la Universidad de Chile. acogiendo los aires de la modernidad, se produjo en 1933, y, en 1934, se llevó a cabo el PRIMER CONGRESO CHILENO DE ARQUITECTURA Y URBANISMO, al que asistió Brunner junto a connotados autoridades y profesionales latinoamericanos. Este Congreso, que también fué primero en su género en Latinoamérica, sentó principios de Urbanismo que abarcaron, en forma pionera, materias de Planificación Territorial. Su programa contempló los temas de la enseñanza de la Arquitectura en Chile; la Planificación Territorial y el Urbanismo; la organización del Servicio de Planificación y de la Dirección General de Urbanismo; las Leyes y Reglamentos de Edificación; la promulgación. en carácter de definitivo, de la Ordenanza General de Construcción y la creación de Ordenanzas Locales como complemento.

Todas estas actividades se llevaban a cabo en el tercer decenio del siglo, cuando la ciudad no veía aún la manifestación de la presión expansiva intensa que provocaría luego la industrialización del país al atraer masivamente a la población rural. En 1930 Chile tenía cuatro ciudades principales que, sumadas, no alcanzaban el millón de habitantes, y las poblaciones rurales y urbanas estaban equiparadas.

En Febrero del año 1938, el PRIMER CONGRESO DE URBANISMO fué celebrado en Valpaíaíso, los profesionales participantes emitieron entonces una declaración de principios que señalaba que la acción reguladora delurbanismo en la vida colectiva debía aplicar en el territorio nacional, o en la ciudad, los planes que le dictara una política superior. Se consideraba necesario también coordinar y organizar las actividades que constituyen la vida colectiva cuyo desarrollo se realizaba entonces en forma desconectada y aún contrapuesta. Por otra parte, se estimaba que la concepción y realización de las obras urbanísticas debian estar en manos de profesionales especializados. Su acción coordinada llevaría a la unidad, esencia de toda expresión artística. Porúltimo, la vivienda, célula primaria de la ciudad, debía ser considerada la base de todo estudio urbanístico constituyendo el problema más importante y apremiante del país.

Los acontecimientos antes evocados, el desarrollo de la actividad académica y la prosperidad del medio profesional, abrieron camino a la necesidad de acceder a una forma de organización académica más integral, propiciándose hacia 1939, junto con la formalización del "COLEGIO DE ARQUITECTURA Y URBANISMO" , la constitución de la "FACULTAD DE ARQUITECTURA Y URBANISMO" en la Universidad de Chile. La Revista de la Asociación de Arquitectos de Chile, "URBANISMO Y ARQUITECTURA" destacaba en su primer número, de mayo de 1939, que el movimiento iniciado en la Escuela de Arquitectura, tendiente a obtener la creación de la "Facultad de Arquitectura y Urbanismo", independiente de Ingeniería había encontrado eco en la Asociación de Arquitectos, en el Instituto Nacional de Urbanismo, y en algunas esferas del Gobierno. donde especialmente el Ministro de Fomento, Arquitecto Sr. Arturo Bianchi, cooperó de manera entusiasta para el logro de estas anhelos.

La Revista mencionada continuó informando en su segundo número, de Julio de 1939 sobre la evolución de la propuesta para nuestra Escuela de Arquitectura en el sentido de la conveniencia de no separar de ella el Urbanismo. Al respecto, el Presidente del Centro de Alumnos de la Escuela de Arquitectura de la época, Sr. Euclides Guzmán Alvarez, manifestaba:

“Etendemos nosotros que una Facultad es un

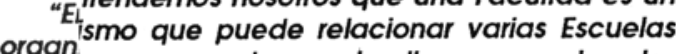
organ aunque cada una de ellas represente solaafines una parte del todo que significa^la Facultad. Asi, el hecho de que haya "Facultad de Arquifectura y Urbanismo" no significa como parece se ha creido, que en nuestra Escuela de Arquitectura se de también al Urbanismo una importancia preponderante. Esto sólo deja la posibilidad, como se ho dicho también, de crear más tarde una Escuela de Urbanismo para Post-Graduados, que abriría un nuevo y extenso campo a la enseñanza de la Arquitectura, quedando nuestra Escuela con su radio de acción actual, mejorando naturalmente su plan de estudios dentro de las posibilidades con que se cuenta".

Hacia 1941, la Escuela de Arquitectura de la Universidad de Chile contaba con un total de 150 alumnos, y se ubicaba en la Facultad de Ciencias Físicas y Matemáticas desde el año 1900. Nuestra "FACULTAD DE ARQUITECTU$R A^{*}$, emergió finalmente en 1944, pero en su denominación oficial no se incorporó el término "Urbanismo". Este hecho fué, al parecer. una expresión del pensamiento tradicional predominante entonces, sobre los alcances del campo de conocimiento y acción de la nueva Facultad, aunque también ha sido señalado como una simple omisión, según consta en una carta del Presidente de la Asociación de Arquitectos de la época, Sr. Ricardo González Cortés, dirigida al Rector de la Universidad de Chile. (1 de Agosto de 1940). La actual denominación de "FACULTAD DE ARQUITECTURA Y URBANISMO", data desde 1966, luego de la creación de MINISTERIO DE VIVIENDA Y URBANISMO, durante el Gobierno del Presidente Frei. 


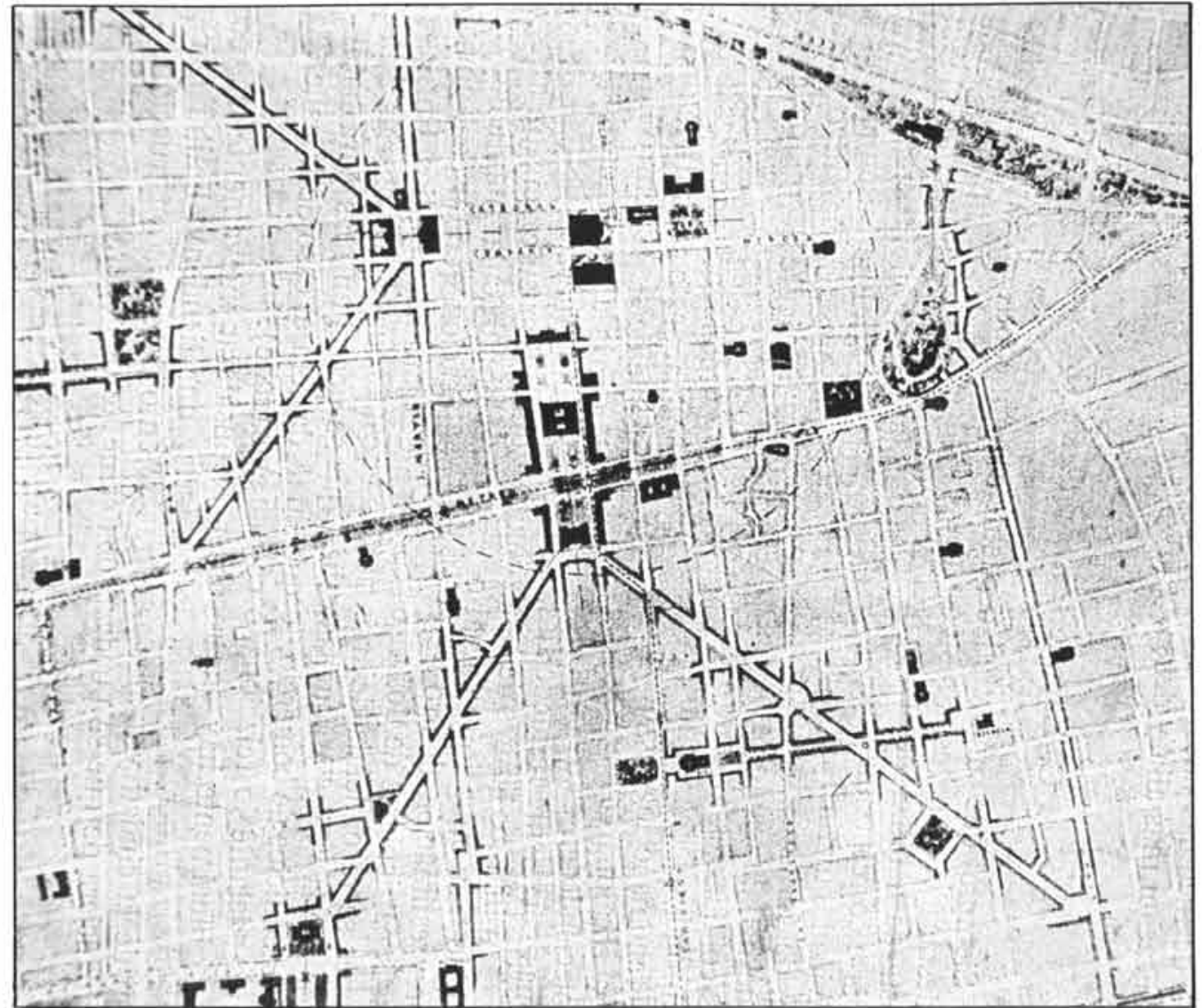

COMUNA DE SAN MIGUEL

$$
\text { ESTUVIE SOBRE EL PLANO DE URBATHZACION }
$$


Institucionalizaclón del campo femóllico del Urbanismo en la "Facultad de Arqullectura" de la Universidad de Chille.

- ntre 1945 y 1948, la Cátedra de Urbanis- mo vivió un período confilcttvo, por L cuanto diversos profestonales se suce dieron en ella, sin lograr consolldar la disciplina que era, por una parte, resistida por el alumnado, y por otra, requerlda en condiclones que satisficieran las dlversas inquietudes surgldas en tomo al Urbanismo de la Post-Guerra.

Sl blen durante los primeros años del siglo $X X$ se había producido el eclipse de las utopias IIterarias y sociales, el disenno urbanístico no dejo de lado la utopía física para el logro de la cludad ldeal, alcanzando una importancia y difusión considerable a partir de los trabajos de Frank Loyd Wright y Le Corbusier. Así como Le Corbusier abogaba por la gran concentraclón urbana. Wright pensaba en su cludad. llamada Broadacre, como caso extremo de dispersión, hasta lograr un hibrido entre campo y cludad. Ninguno de los Arquilectos cltados, y cuya Influencla alcanzó la segunda mitad del siglo, se detuvieron a analizar los aspectos económicos, sociales ni políticos, pensando que la restauración del amblente material traería como consecuencia el naclmiento de instituciones idóneas para su goblerno.

Al respecto el impacto en nuestro medio. en la visión general retrospectiva de una exalumna de nuestra Facultad parece elocuente:

"La nuestra fue una generaclón cuyo camino se inicio en un mundo convulsionado por una guerra mundial que, por una parte, puso en jaque valores que parecian indestructibles, y por otra, abrió espe ranzas de un mundo mejor. Sentimos entonces un fuerte compromiso con esta apertura y nos parecía posible sublimarlo desde nuestra profesión; estudiabamos Arquitectura no sólo para construir bellos edificios y viviendas, sino para plantear a través de ellos nuestra visión de un mundo más armónico que diera opción a todos los hombres, de una vida más plena. Nos parecía vivir en una arista que separaba este mundo que se autodestrula de aquel otro mundo que estaba por construirse y al cual adheriamos con todo el entuslasmo de nuestrajuventud. Ast aumentaba para nosotros el magnetismo de "lo nuevo" y adheriamos con todos nuestras tuerzas al movimiento moderno. Descubriamos los postulados del CIAM y aspirábamos a cludades de trazos geométricos sobre un plano verde... que serian contempladas y vividas por ese hombre abstracto que tan concretamente dlbujaba le Corbusler en sus planos. Inconscientemente nos remitiamos a $\theta$ y a él referiamos alturas, anchos y largos, utilizando los más sofisticados métodos para la aplicación de trazados reguladores. Hablábamos muy sinceramente de compromisos con EL HOMBRE sin darnos cuenta que era esa imágen de hombre con la que nos comprometíamos y no precisamente con aquél que compartiamos nuestra vida cotidiana. Todo cuanto proyectábamos en la Escuela eran espacios capaces de ser reconocidos y distrutados por hombres como aquél que nos mostraba el canon. de medidas perfectas y senslbilidad "maderna". En esa época ávida de "modernidad" detestábamos todo aquello que en el entorno que nos rodeaba se apartara de esa imagen y nos proyectóbamos a un futuro que demandaria de nosotros encargos a una escala capaz de transformarlo. Los primeros pasos en la vida profesional fueron moderando nuestro impetu y aprendimos que los encargos provenian de entes muy concretos, de instituciones o de personas que, al igual que nosotros, convivian en esa larga faja de tierra de loca geograffa y escasos re cursos. Lentamente fuimos aceptando las diferenclas que nos marcaban como Arquitecto de este tercer mundo en el que todo estaba por hacerse. pero en el que no se tenía claro, nl que, ni cormo, nl 
cuando podia hacerse" ... "nos costó aceptar que nuestro destino no era el de repetir grandes gestos sino inventar aquél que, al multiplicarse, construyerauna gran figura". (Arqta. Angela Schweitzer, 1987).

Desde 1946 y hasta inicios de 1952, Muñoz Maluschka se desempeñó como Profesor de Urbanismo para los niveles de tercero, cuarto y quinto año de la carrera de Arquitectura en nuestra Facultad. Inspirado en sus estudios de Urbanismo realizados en Baja Sajonia, Alemania, en su experlencia como colaborador y contraparte de Brunner y, en su experiencia como impulsor de una notable cantidad de proyectos de Planificaclón Urbana Regional en tanto Jefe de la Sección de Urbanismo del Ministerio de Obras Públlcas, dictaba un curso matizado por las tendencias cientíícas que los racionalistas alemanes desarrollaban en materia de análisis, proyectos y administración urbana.

La experiencia de Alemania había mostrado ya, desde fines del siglo XIX, que la ciudad podía ser una verdadera máquina productiva de excepcional compacidad y coherencia. donde la coordinación entre el poder público y el privado podía ser realidad. Los resultados en lo referido a acondicionamiento de los ríos y muelles portuarios, a las diversas obras civiles para la producclón y distribución de energías. a los equipamientos urbanos de todo tipo, a los sistemas de transporte subterráneos y de superficle, y otros, habían difundido una práctica urbanística altamente tecnificada, y que había dejado en segundo plano los objetivos estéticos del Urbanismo. Tal vez por ello, la enseñanza de Luis Muñoz, fué poco considerada por muchos de sus alumnos quienes, en gran número, no concurrían a sus clases e incluso le criticaban sin haber conocido la profundidad. riqueza y modernidad de sus conceptos. El Profesor Muñoz, alejándose de las utopías asociadas a las formas urbanas, se habian Inclinado por la apreciación del fenómeno urbano y regional en complejidad y profundidad.

Desde 1945, no obstante, la renovación del espíritu y estructura pedagógica de la Facultad de Arquitectura había ido abriendo cauce al desarrollo de la mentalidad clentífica en los estudiantes y en el personal docente. La primera expresión de esta nueva mentalidad fué la organización de los dos primeros cursos de la Escuela de Arquitectura con un fuerte acento en el análisis, pretendiendo formar en el alumnado una actitud viva frente a la enseñanza recibida, y un método básico para la posterior creación arquiltectónica y urbanística. Muy pronto el ejerciclo cotidiano de esta metodología logró poner en evidencia los nexos esenciales entre la Arquilectura, el Arte y la Técnica, y de ellos con la vida social y económica, destacando problemas de necesaria investigación y clasificación.

Hacia fines del cuarto decenio del siglo, la situación habitacional chilena había comenzado revelarse con dramática crudeza. Las poblaciones insalubres habian rodeado súbitamente a Santiago y otras cludades industriales, provocando las más dispares opiniones sobre la solución que ellas reque rían. Asi, el déficit de habitaciones y de edificios para los servicio sociales y culturales, el creciente desequilibrio de las zonas urbanas y rurales, el precario desenvolvimiento de la técnica aplicada a la edificación, el abandono del patrimonio historico de la Arquitectura nacional, fueron algunas de las razones determinantes que llevaron, a plantear la Idea de crear organismos especializados que, junto con ofrecer una enseñanza superior especializada. cooperaran en la solución de tales problemas. Tanto el personal docente de nuestra Facultad en la época, como el alumnado, no podían satisfacer. dentro de la organización escolar, las amplias y variadas exigencias de estudio que una nueva metodología entrañaba, y cuyos resultados aparecían indispensables para cimentar una mejor enseñanza.

Porotra parte, una segunda expresión dela nueva actitud científica de la Facultad de Arquitectura, fué la creación en 1951, de la Cátedra de SEMINARIO DE INVESTIGACION en el sexto año de la Carrera. Paralelamente, se iniciaba el funcionamiento experimental de los INSTITUTOS como entidades orgánicamente independientes de la Docencia, aunque estrechamente relacionados con ella a través de los mencionados Seminarios de Investigación.

Las necesidades derivadas de la nueva realidad configurada, se vieron satisfechas - cuando en Febrero de 1952, el Consejo Universitario dio la aprobación oficial a los nuevos institutos de la Facultad creados por el Decano Héctor Mardones Restat, estableciendo su rol frente a las exigencias de enseñanza supe rior especializada que la realidad del país demandaba en el orden cultural y material.

EL INSTITUTO DE VIVIENDA, URBANISMO Y PLANEACION se creó de esta forma, paralelamente a los Institutos de HISTORIA DE LA ARQUITECTURA y de EDIFICACION EXPERIMENTAL. EI INSTITUTO DE ESTABILIDAD EXPERIMENTAL habí sido fundado en 1940, siendo Decano don Gustavo Lira Manso, cuando la Universidad de Chile contaba solamente con tres institutos de investigación; este número llegaría a sesenta. veinte años después.

El Instituto de Vivienda, Urbanismo y Planeación representó en la Universidad de Chile y en la organización universitaria nacional, la primera forma de Institucionalización académica designada para el estudio de los fenómenosurbanos-regionales en forma integral. A partir de la labor de dichos institutos se ampliaría y profundizaría los campos de conocimiento y acción de la Facultad sistematizándose las líneas teóricas y técnicas de modo de complementar un proceso docente que, hasta entonces, se habia centrado principalmente en la tramitación deloficioy en la experiencia práctica.

2. ESTUDIOSOBRELAESTRUCTURA ARQUITECTONICADELA CIUDAD DE SANTIAGO DE CHILE, DE K. BRUNNER. (Orig nal Esc. 1:5.000). La necesidad de impulsar el desarrolo urbano del sector central, al sur de la Alameda. llevó a Brunnera proponerla apertura denuevas grandes a terias y de una edificación densa para esa extensa zona. (en: Brunner, 1932, op. cit.).

3. ESTUDIO SOBRE EL PLANO DE URBANIZACION DELACO MUNA DE SAN MIGUEL, DE K. BRUNNER, (Original ESC. 1:5.000). Brunner puso el énfasis en el sistema de calles y avenidas, basándose en el principio de a subdivisión estricta en: arterias de tránsito en ambas direcciones; calles de tránsito secundario o de acceso a las prime ras, y calles residenciales de diversas categorías. (en: Brunner, 1932, op.cti.)

4. EDIFICIO EX PALACIO DE LA REAL ADUANA CON EL PORTAL QUE PROPUSO K. BRUNNER EN EL PRIMER PISO. Esta idea tuvo por finalidad eliminar la estrechez a costado Norte del edificio, en la necesidad de reforzo el eje Merced-Compañia, como eje principal del centro, de Oriente a Poniente. El alcalde P. Mekis retomó la idea, que fué incorporada por el Arqto. F. Riquelme o proyecto de restauración realizada en los años 1970. (en: Brunner op. cit.)

5. PROYECTO DE 2.000 VIVIENDAS POPULARES EN VIENA DE K. BRUNNER, 1928, (en: K. H. Brunner, 1939, op. cit. proceso en: U. Fotografía, Archivos y M. Audiovisuales de la U. de Chile).
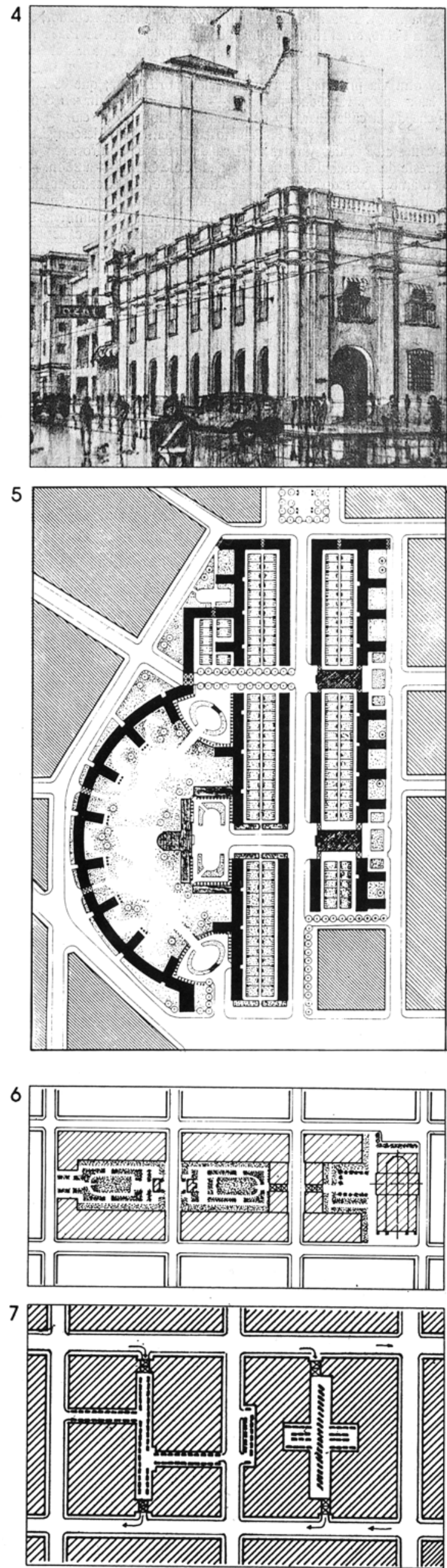

6.. USO DE SUELO EN EL INTERIOR DE LAS MANZANAS PROPUESTO PORBRUNNER. Ejemplo esquemático de uso de suelo en árecs verdes de uso público vecinal. Brunner recomendó reglamentar estos usos para un móntmo aprovechamiento del suelo. (Dibujo de E. Lyon a partir aprovechami 1932 dep. cit.).

7. USO DE SUELO EN EL INTERIOR DE LAS MANZANAS PROPUESTOPORBRUNNER. Ejemplo esquemático de uso de suelo en estacionamiento de vehiculos. Brunner recomendó reglamentar estos usos para un máximo aprovechamiento del suelo. (Dibujo de E. Lyon a partir de Brunner, 1932, op.cit.). 


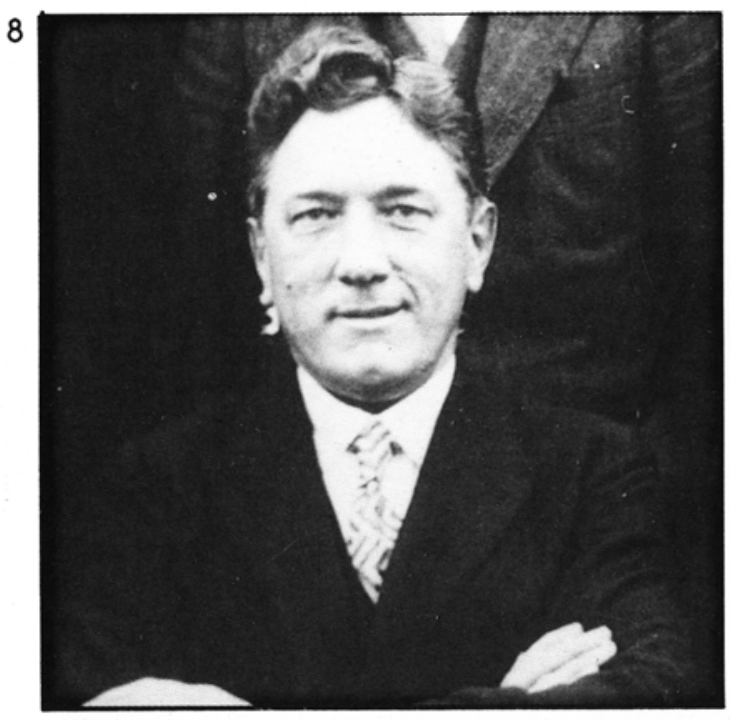

ARQUITECTO ALBERTO SCHADE POHLENZ, PrOfesor fundador del Primer Curso de Urbanismo en la "Escuela de Arquitectura de la Facultad de Ciencias Fisicas y Matemáticas" de la Universidad de Chile.

A

Iberto Schade, nació en Santiago el 28 de Julio de 1822. Hizo sus estudios secundarios en el Instituto Nacional, y superiores en la Universidad de Chile. Obtuvo su título de Arquitecto en 1907. Trabajó en el Departamento de Arquitectura de la Dirección de Obras Públicas desde el año 1904 y hasta 1918. Durante los años 1911 y 1914, permaneció en Francia comisionado por el Gobierno, para hacer estudios sobre Arquitectura y Construcción. Su vocación docente por esta última especialidad le motivó escribir, algunos años después, el libro ARQUITECTURA INDUSTRIAL. (U. de Chile), que constituyó un valioso manual de construcción, cuya tercera edición se realizó en 1954.

Participó activamenteen 1923,como miembro del Comité Ejecutivo, en el Segundo Congreso Panamericano de Arquitectos, donde predominó el interés unánime por resolver los problemas del desarrollo urbano. El estudio racional de la vivienda americana, y el desarrollo de la enseñanza en Urbanismo, con la creación de las Facultades de Arquitectura, formaron parte también de los temas tratados en el Congreso. En 1930, se desempeñó como delegado oficial de la Universidad de Chile al Congreso de Arquitectos de Río de Janeiro.

Schade fué Presidente de la Asociación de Arquilectos y miembro del Instituto de Ingenieros. Entre sus diversas obras de Arquitectura construídas en Santiago, destacaron la Escuela Arquitectura, en la plaza Alonso de Ercilla de Santiago; el edificio de renta, de don Marcial Martínez de Ferrari, ubicado en las calles de San Antonio con Monjitas; el edificio de renta ubicado en Miraflores esquina Ismael Valdés Vergara (sur-oriente), para el cual trabajó en conjunto con Oyarzún Phillipl, el edificio 'La Mundial", realizado con este mismo socio; la residencia Schleyer, construída en 1926, en Providencia esquina de Nueva de Lyon, para lo cual el equipo incorporó también a Muñoz Maluschka, además de numerosos edificios particulares para residencia.

En materia de Urbanismo, Alberto Schade es autor del PLANO DE SANTIAGO: "CONTRIBUCION AL TRAZADO RACIONAL DE AVENIDAS DIAGONALES Y TRANSFORMACIONES DE LA CIUDAD", presentado en Septiembre de 1923, cuyo original, Escala 1:5.000, pertenece al patrimonio de nuestra Facultad. En 1929, partici- pó en una Comisión integrada, además, por Muñoz Maluschka y los Ingenieros, Jorge Alessandri y Francisco Mardones, en el estudio "BASES PARA UN PLAN REGULADORDE SANTIAGO", ciudad que contaba en ese momento con casi 700.000 habitantes.

El Curso de Urbanismo fundado por Schade a partir de 1928, cuando contaba con 47 años de edad, era en la próctica un curso de URBANISTICA, originado en sus amplios conocimientos técnicos. Sus estudios sobre las infraestructuras de agua potable y alcantarillado de la ciudad, asociándolas a las densidades de población y su evolución, llevaron al Profesor Schade a relacionar las disciplinas de rigor en materia de urbanización, con las ciencias sociales. Su visión social de la Arquitectura y el Urbanismo es tal vez lo más destacable de su enseñanza, que en materia de ejercicios en el curso de Urbanismo, se centraba en el tema de los Planes Reguladores.

No obstante, se ha destacado en algunos escritos que el curso de Alberto Schade se inspiraba principalmente en los principios y técnicas desarrollados por Camilo Sitte, autor de "Der Städte-Bau nach seinen Künstlerischen Grundsātzen", ("El Urbanismo según sus Principios Artísticos"), escrito en 1889. Las materias abordadas por Sitte, fueron en efecto tratadas por Schade, pero formando parte de una de las unidades de materia del curso, la unidad denominada "Estética de la Cludad". Sitte, llamado el "padre del urbanismo moderno",y cuya formación en Viena incluyó además de la Arquitectura, la Historia del Arte, la Anatomía, la Filosofía y la Música, fué quien inició el camino del conocimiento sintético de las relaciones que ligan los diversos aspectos de los problemas urbanísticos, como también el conocimiento de las influencias múltiples que ellos ejercen.

Antes, cada materia se trataba aisladamente. en orden cronológico, partiendo de un punto de vista exclusivamente estético arquitectónico para considerar los problemas urbanísticos. Sitte señalo la importancia de la percepción del espectador moderno y del período histórico en cuestión. Su reflexión, a partir de conceptos artísticos abstractos. más que de detalles específicos de la realidad. planteo una forma de análisis absolutamente moderna: insistió en la necesidad de una concepción tridimensional de la ciudad, en la búsqueda de la continuidad de los efectos espaciales en el tejido urbano, y en la definición de continentes y contenidos, en este ámbito. También comprendió que los espacios públicos estaban llamados a otorgar el carácter de una ciudad y que por ello debían presentarse como secuencias de espacios atractivos y útiles. La consideración de la vegetación como conjunto de elementos tridimensionales que forman parte de la estructura espacial urbana. además de aportar valores higiénicos, decorativos y de recreación, y, en general, la concepción del mundo natural y del mundo artificial como un todo. señalaron un nuevo camino en materia de Urbanismo, cuando sólo faltaba una década para la llegada del siglo XX.

En Chile, Schade difundió tamblén estos conceptos, que, si bien habían sido escritos en Europa cuarenta años antes, significaron finalmente una verdadera revolución del pensamiento y quehacer profesional a nivel mundial.

La Escuela de Ingeniería y la Escuela de Arquitectura de nuestra Universidad, contaron a Alberto Schade entre sus más distinguidos Profesores, obteniendo los frutos directos de su destacada labor docente durante 22 años. En el año 1931, y por razones ajenas al quehacer universitario, Schade fué alejado de sus labores docentes. Su obra aún no ha sido estudiada.
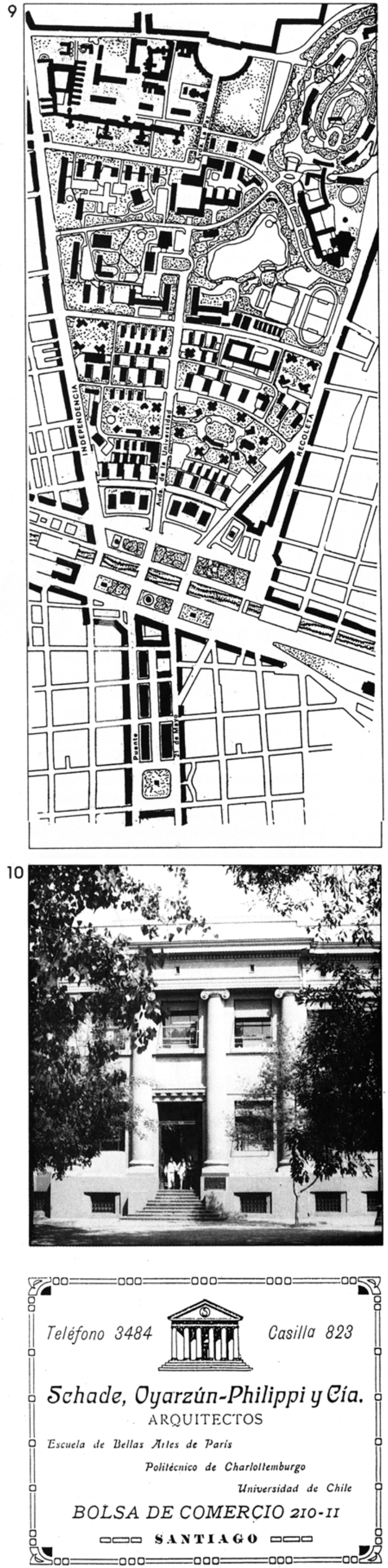

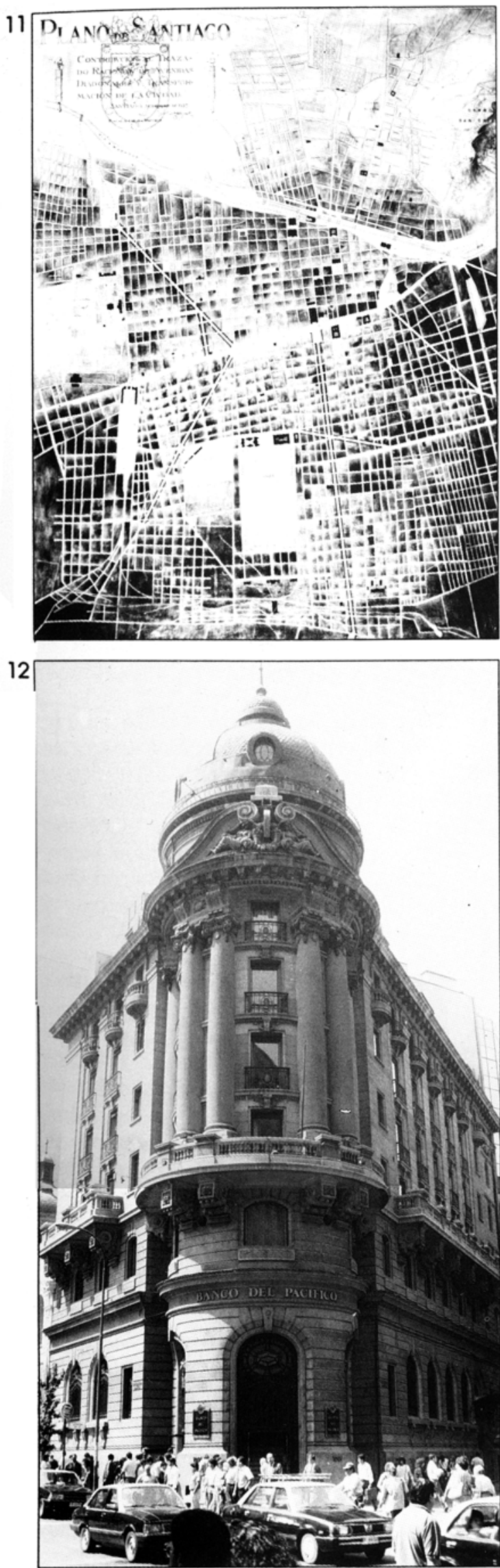

8. ARQUITECTO ALBERTO SCHADE POHLENZ (U. de Chile, F.A.U., Archivos Fotográficos de 1992; Proceso en: U. Fotografia, Archivo y M. Audiovisuales de laU. de Chile)

- ANTEPROYECTO PARA LA UNIVERSIDAD DE CHILE REMODELAMIENTO URBANO DE SANTIAGO, DE RODULFO OYARZUN YELSA FUENTES DE OYARZUN. Se proponic la emadicación de la Vega y su reemplazo por la Ciudad Universidad, completada con grandes conjuntos de vivienda destinados a los funcionarios, académicos y estudiantes. Amplias zonas verdes y espacios deportivos y de recreaclón permifín romper la cuadrícul ur y de recreaclón permific rom la ciudad desde Plara de Amas hacia el Norte. (Dibujo de E. Lyon, partir de: U. del Bio Bio, 1988, op. cit.).

0. ESCUELA DE ARQUITECTURA DE LA UNIVERSIDAD DE CHILE, DE ALBERTO SCHADE. (Foto de R.Chandía 1991. U. Fotografia, Archivos y M. Audiovisuales de la U. de Chile).

11. PLANODESANTIAGO: CONTRIBUCIONALTRAZADO RACIONAL DE AVENIDAS DIAGONALES Y TRANSFORMATOSCHADE. (Orignal Esc. 1:5.000.U. Fotografia. Archivos y M. Audiovisuales de la U. de Chile)

12. EDIFICIOLA MUNDIAL, de RODULFO OYARZUN Y ALBER TO SCHADE. (Foto de R. Chandía, 1991. U. Fotografia Archivo y M. Audiovisuales de la U. de Chile).

13 ARQUITECTORODULFO OYARZUN PHILIPPI (U. del Bí Bio, op. cit.: Proceso en: U. Fotografia, Archivo y M. Audiovisuales de la U. de Chile).
ARQUITECTO RODULFO AMANDO OYARZUN PHILIPPI, Profesor de la Cátedra de Urbanismo a la partida del Profesor Karl Brunner.

1 odulfo Oyarzún Philippi fué un hombre multifacético, destacándose como Arquitecto, Urbanista, Pintor, Escultor, y Educador en las líneas de las Artes Plásticas, la Arquitectura y el Urbanismo. Había nacido en 1895, y realizó sus estudios básicos y medios en el Instituto Nacional. A los 17 años se encontraba cursando un semestre en las especialidades de Construcción e Historia del Arte en el Politécnico de Charlottenburgo, en Berlín.

En 1927, ya titulado de Arquitecto, realizó su segundo viaje a Europa dondé visitó durante dos años las grandes capitales, estudiando Arquitectura Moderna en la Academia de Bellas Artes de Viena con el maestro C. Holzmeister, asistiendo también en Viena al "Seminario de Urbanismo" del profesor Karl Brunner y al curso de postgrado "Introducción al Urbanismo" del Profesor O. Iltz. En este viaje, tomó también cursos de fotografía, y de canto con el maestro Obers Stetter.

A su regreso a Chile asumió una posición de vanguardia en la búsqueda de la modernización de la enseñanza y práctica de la Arquitectura, proponiendo entre otras cosas, la contratación del Dr. Brunner para la enseñanzay asesoría del Gobierno en materia de Urbanismo en 1929. En la Universidad, Oyarzún Philippi fué invitado a servir la Ayudantía en la Cátedra de Brunner. También en 1929, cofundó el Instituto Nacional de Urbanismo, del cua fué Presidente en 1934.

En 1932, a la partida de Brunner y hasta 1946, Rodulfo Oyarzún asumió la Cátedra de Urbanismo, creando un Taller de Urbanismo para tratar conjuntamente los aspectos teóriros y prácticos. En 1934, siendo Presidente de la Asociación de Arquitectos, organizó y presidió el Primer Congreso Nacional de Arquitectura y Urbanismo, impulsando desde estas funciones, diversas campañas para el bienestar de la comunidad.

En 1937, viajó nuevamente a Europa, profundizando sus estudios en Urbanismo y Planeamiento. Cumplió una misión ad honorem del Ministerio de Obras Públicasy, por segunda vez logró contratar al Dr. Brunner.

A raíz del terremoto de 1939, el Instituto de Urbanismo que presidía, propuso la creación de un MINISTERIO DE RECONSTRUCCION, planteando la necesidad de planificar con criterio científico la reconstrucción de las ciudades devastadas.

Hacia fines de la década de los años 30 había realizado el PLAN REGULADOR DE ALGARROBO, y los anteproyectos de los PLANES REGULADORES DE CONCEPCION, CHILLAN Y LOS ANGELES, a los que se sumó, en 1958, el anteproyecto deIPLANREGULADORDECONS TITUCION. Con su esposa, Elsa Fuentes, desarrolló un proyecto de CIUDAD UNIVERSITARIA PARA LA UNIVERSIDAD DE CHILE, que se ubicaría junto al Río Mapocho, entre las calles Recoleta elndependencia, constituryendo estaproposición uno de sus más significativos aportes teóricos al desarrollo de Santiago.

Inspirado en el pensamiento de Brunner participó de la definición del Urbanismo mo derno a partir del tema de la vivienda, cuyos problemas debian ser coordinados en el mar co general del desarrollo económico y social del país. En su concepción, la coordinación de las múltiples factores de la existencia humana

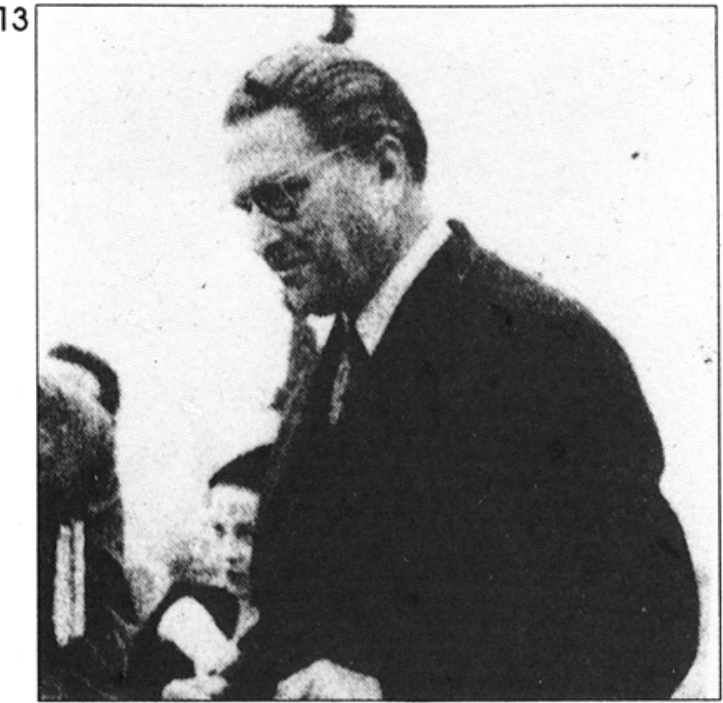

economizando esfuerzos y dirigiéndolos hacia la realización de los objetivos de bien común deseados, era misión fundamentalmente de esta disciplina.

Inquieto por la diversidad de estilos en las áreas residenciales, evocaba en sus escritos laspoblacionesnuevaseuropeas consus casas "tipo standard", según el nuevo ideal. El problemas del tránsito en la ciudad no estaba ausente en sus reflexiones señalando la urgencia de aumentar y jerarquizar las vías, de adecuar el horario de trabajo para evitar la congestión y, de aplicar una política racional de densidad urbana

Denunciaba, por otra parte, la degradación de las ciudades, la especulación del suelo el desarrollo innecesario de la construcción en altura, el alto costo de la ampliación de los senvicios básicos por la extensión desmesurada de la ciudad y la carencia de espacios libres verdes en ella. La ausencia de conciencia generalizada sobre la necesidad de impulsar avances en materia de Urbanismo en Chile, le motivó a realizar numerosos encuentros, conferencias, y publicaciones en los principales periódicos del país.

Rodulfo Oyarzún, adhirió también a las iniciativas de la primera mitad del siglo, en relación con la creación de un sistema de transporte rápido masivo, observando, además. que la ciudad crecía sin orientación y que las solas iniciativas puntuales provenientes de las autoridades comunales para resolver los problemas de Santiago eran insuficientes. Por ello propuso la creación del cargo de "Alcalde Mayor" para el conjunto de Comunas del Gran Santiago.

La importante labor desarrollada por el Profesor Rodulfo Oyarzún dela cual citamosen estas líneas sólo algunos momentos, fué reconocida al otorgársele diversas distinciones: MEDALLA DE ORO, en 1968 (Ministerio de V1vienda y Urbanismo); PREMIO NACIONAL DE ARQUITECTURA, en 1974 (Colegio de Arquitectos de Chile); ACADEMICO MAS DESTACADO. en 1977 (Universidad Técnica del Estado. Concepción); PROFESOR EMERITO, 1980 (Facultad de Arquitectura y Urbanismo de la Universidad de Chile); CIUDADANO DISTINGUIDO 1981 (I. Municipalidad de Concepción), y el nombramiento de PROFESOR EMERTO, por la Facultad de Arquitectura y Construcción de la Universidad del Bío-Bio en 1982 (Fte.: Universidad del Bío-Bío, op.cit., 1988). 


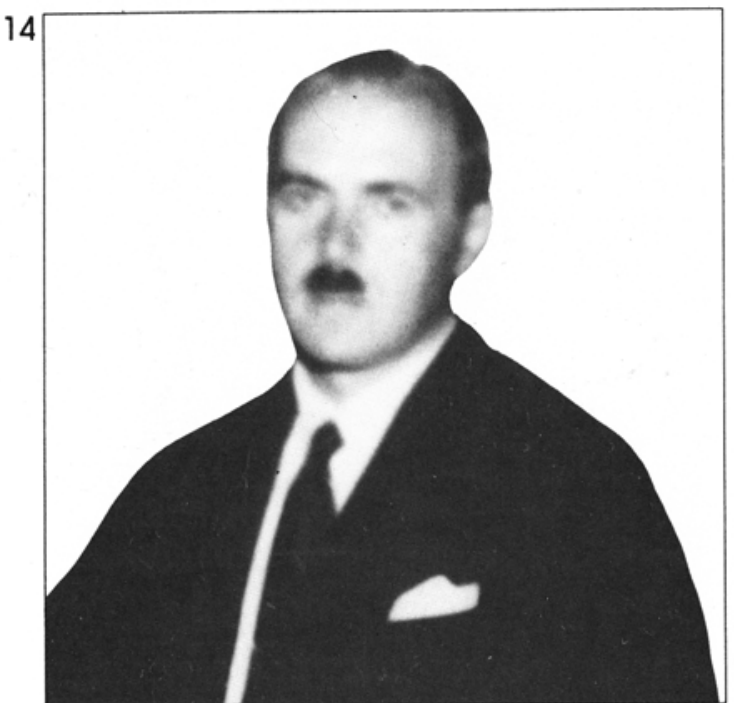

ARQUITECTO DR. KARL HEINRICH BRÜNNER VON LEHENSTEIN. Profesor fundador del Primer Seminario de Urbanismo realizado en Chile y América Latina.

K arl Brunner llegó a Chile en 1929, a la edad de 42 años, contratado como Asesor del Gobierno y Profesor de la Universidad de Chile. La enseñanza regular que impartía en Viena se basaba en su SEMINARIO DE URBANISMO, iniciado en 1924-25 en la Facultad Nacional de Arquitectura de Viena, con asistencia de estudiantes y profesores de diversos lugares del mundo. Este plan de estudio tuvo gran éxito en el Congreso de Urbanismo de Heilderberg, Alemania, en 1928. año en que fué implantado en varias universidades europeas.

Contando en Chile con la valiosa colaboración de Oyarzún Philippi, profesor de la Escuela de Arquitectura (34 años), y Muñoz Maluschka (33 años), Jefe de la Sección de Urbanismo de la Dirección General de Obras Públicas, este Seminario fué creado en Santiago, constituyéndose en un curso intensivo de preparación de la primera generación de Urbanistas realizado en Chile a nivel de graduados. En el Seminario el Dr. Brunner, trataba la materia en tres capítulos:

\section{I.- ASUNTOS POLITICOS ECONOMICO-SOCIA-} LES Y LEGALES: Política de Desarrollo Urbano: Política Vial y de Comunicaciones Urbanas: Fomento de la Habitación Popular; Higiene Social Urbana; Fisiología y Sociología Urbanas; Legislación de las Construcciones Urbanas y de Urbanización, Estudios sobre Avalúos, Impuestos, y Financiamiento del Desarrollo Urbano.

II.- ASPECTOS DE LA TECNICA DE LAINGENIERIA EN SU APLICACION A LAS CIUDADES: Urbanización de Terrenos, Trazado y Perfiles de Vías; Vías Subterráneas e Instalación de Subsuelo: Agua, Alcantarillado, Conducciones (Urbanismo Subterráneo); Construcción de Habitaciones y Poblaciones; Areas Verdes, Parques, Paseos Públicos, Canchas de Deporte, etc.; Estaciones y Líneas de Ferrocarriles, Ferrocarriles Metropolitanas (elevadosy subterráneos). Tranvías, etc.; Planificación de Cludades, Regularización y Ensanches.

III. LOS ASPECTOS ESTETICOS, ARQUITECTONICO Y ARTISTICOS: Creación Plástica de la Ciudad Moderna: a) Construcciones Monumentales y Conjuntos de Edificios; b) Callesy Plazas;

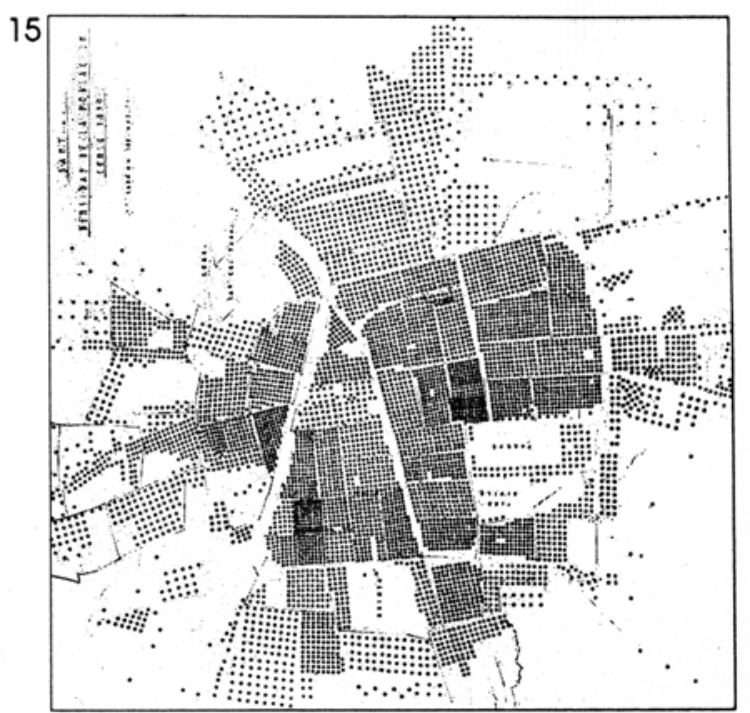

c) Parques (Arquitectura Paisajista); d) Estructura Arquitectónica de Poblaciones y de las Ciudades; Historia del Arte Urbano; Conservación de Monumentos y Parques.

Entre los conceptos que Brunner desarrollaba en los capítulos citados, estaba el de la vivienda vista como la célula primaria de la ciudad. Su tesis, siguiendo la POLITICA DE CONSTRUCCION, indicaba que la nación debía, en primer lugar, establecer un PLAN GENERAL DE DESARROLLO TERRITORIAL que cubriera todo el país. El desarrollo territorial sería la manifestación física del desarrollo económico y social del país. A este Plan Básico debía seguir la formulación de una POLITICA DE VIVIENDA, correspondiendo desarrollar un PROGRAMA NACIONAL DE CONSTRUCCION HABITACIONAL. Brunner consideraba que la Política de Construcción era una de las palancas más poderosas para fomentar el bienestar de los pueblos modernos, y ella estaba íntimamente ligada los problemas económicos y sociales y se consideraba desencadenante del desarrollo Industrial y la explotación de los recursos naturales.

Esta tesis, en la que buscaba el manejo racional y científico del desarrollo urbano. causó gran entusiasmo entre sus alumnos, quienes eran advertidos, no obstante, del riesgo de perderse en el campo de las realidades. si además de su preparación teórica o académica, el Urbanista no estaba preparado para enfrentar los factores adversos a su labor: el desequilibrio social, el individualismo egoista, la especulación, el comercialismo, el oportur nismo, las utopías, la tramitación, las reorganizaciones, los tropiezos legales, el diletantismo. la imitación, la autocracia, la inconstancia, los comentarios y polémicas, la incultura, las importunidades $\theta$ insipideces, el desaseo, y la geometría irreflexiva, entre otros.

El impacto que causó en el medio profesional el Seminario de Brunner tuvo, entre otros frutos, la renovación del pensamiento sobre el hecho urbano, señalándose la necesidad de un conocimiento más sistemático de la realidad para acciones mejor orientadas a la resolución de los problemas. La creación de las Facultades de Arquitectura en Latinoamérica se volvía de esta forma un requerimiento indispensable para la enseñanza del Urbanismo. Después de concluir su fructífera labor como Maestro, y Urbanista en Chile, Brunner fué llamado por la Municipalidad de Bogotá, Colombia, para organizar y dirigir el Departamento de Urbanismo de dicha ciudad y fué designado luego para desempeñar la nueva Cáte- dra de Urbanismo en la Facultad de Arquitectura de la Universidad Nacional de Colombla. La misión en Chile motivó a Karl Brunner a publicar su libro MANUAL DE URBANISMO edltado por el Concejo Nacional de Bogotá en el año 1939.

Brunner volvió posteriormente a Austria y. en los años cincuenta, fué Director General de Obras Públicas de la ciudad de Viena hasta su muerte, en 1960. A fines del año 1953, tuvo ocasión de conocer el proyecto "Santiago una Gran Ciudad" en el cual, su autor, el Arqto. Juan Parrochia, propuso un plan intercomunal para Santiago, Brunner aportó numerosas y valiosas ideas y posibilidades a ese plan, que fué una de las bases del Plan Regulador Intercomunal de Santiago, aprobadoen 1958-1960.

Luego de la partida de Brunner de Chile. Muñoz Maluschka logró concertar a los Departamentos de Ferrocarriles, Vialidad y Obras Sanitarias para llevar adelante importantes reformas urbanas y proyectos desarrollados por el profesor vienés, donde destacan la remodelación Parque Bustamante, la apertura de la Diagonal Oriente, el proyecto de remodelación para la creación del Barrio Cívico, y la apertura de la Avenida Bulnes, entre otros.

14. ARQUITECTO KARL H. BRUNNER VON LEHENSTEIN (U. de Chile, F.A.U.,Archivos Fotográficos de 1929: Proceso en: U. Fotografía. Archivo y M. Audiovisuales de la U. de Chile).

15. EXPEDIENTE URBANO DE SANTIAGO: DENSIDAD DE POBLACIONEN 1930. K.H. BRUNNER, Cada punto represenBLACIONEN 1930.K.H.BRUNNER. Cada punto representa 100 habitantes, demostrándose, entreotros, la formación de un barrio comercial en el centro de la ciudad. y también, parte de la nueva metodología de and́lisis propuesta por Brunner, (Dibujo deA.Riedel, en: Brunner. 1932, op. cit.).

16. PLANO OFICIAL DE URBANIZACION DE LA COMUNA DE SANTIAGO, DE K.H. BRUNNER, 1934 (Reproducción a partir de Orginal Esc. 1:5.000, por (Rentieza del Prof. partir Alberto Gurovich W.). El deseo de Brunner de transformar Santiago en una ciudad agradable se aprecia en sus proposiciones a escala de micro-urbanismo, donde plazuelas y espacios de interés local, colaboran a la conformación de la unidad residencial con un criterio realistaen su aplicación donde era posible, en la estructura urbana. 


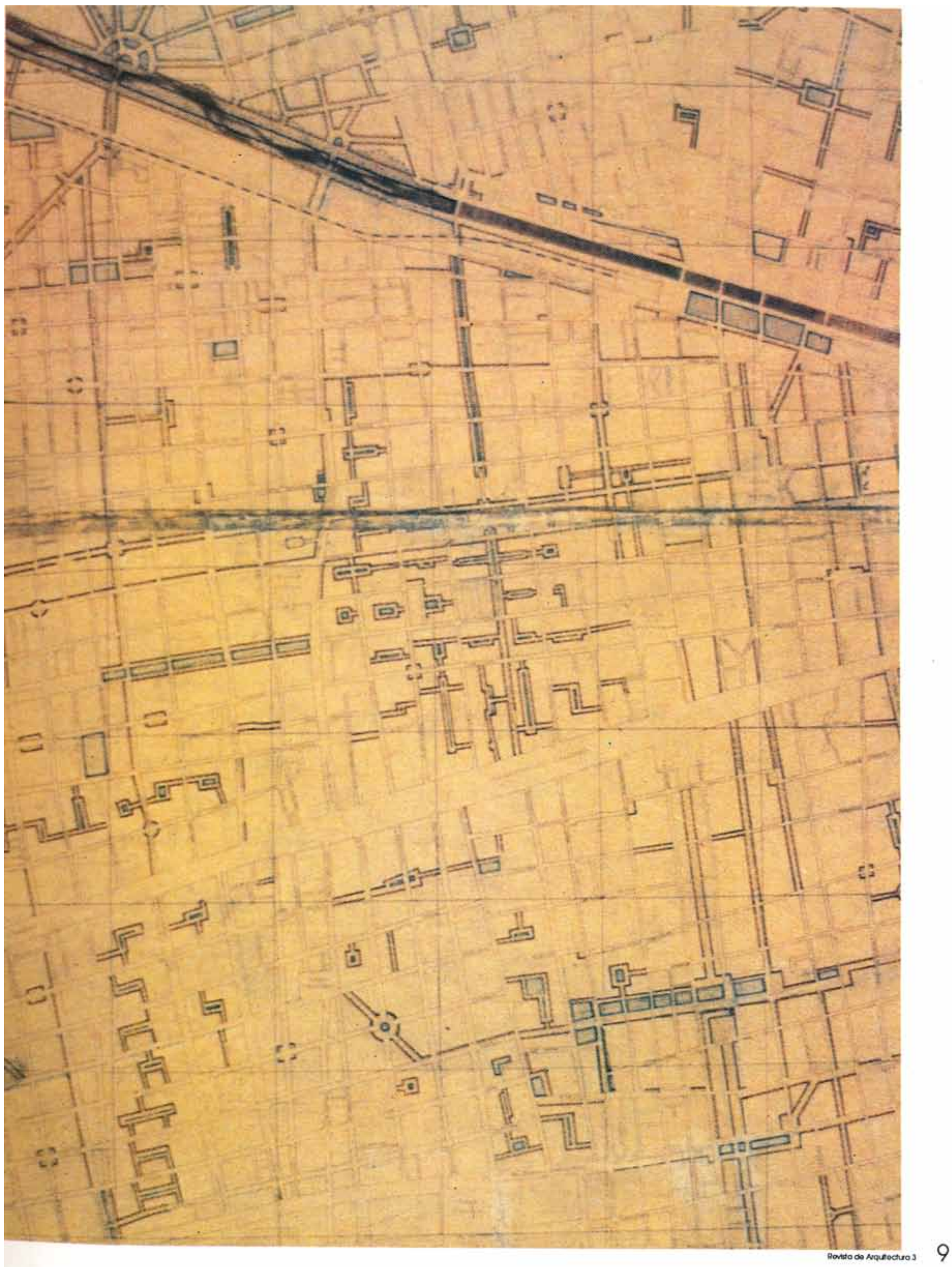



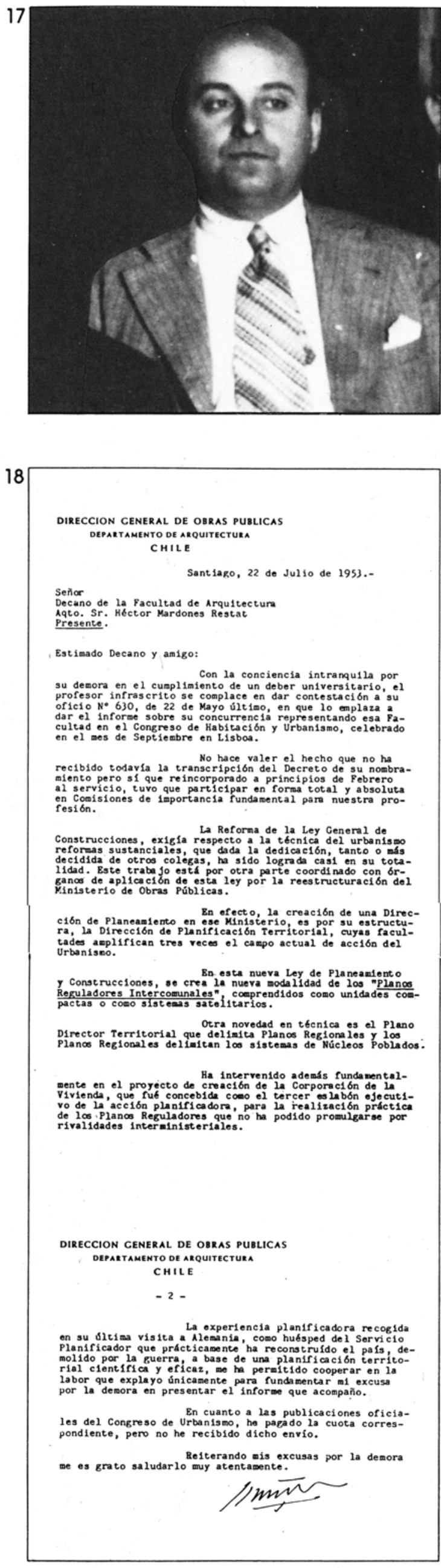

17. ARQUITECTO LUIS MUÑOZ MALUSCHKA, U. de Chile, F.A.U., (Archivo fotogrófico, década de los años 1920: Proceso en: U. Fotografía, Archivos y M. Audiovisuales de la U. de Chile).

18. CARTA DE L. MUÑOZ, al Decano H. Mardones (En: Archivo Histórico del Depto. de Urbanismo, F.A.U., U. de Chile).
ARQUITECTO LUIS ENRIQUE MUÑOZ MALUSCHKA, Profesor de Urbanismo en la "Facultad de Arquitectura" de la Universidad de Chile, y Precursor de la Planificación Territorial en Chile.

uis Muñoz Maluschka nació en Valdivia en 1896, hizo sus estudios en el Instituto Nacional y se tituló de Arquitecto en la Universidad de Chile en 1920. Ejerció su profesión libremente en Santiago y Valparaíso. Especializado en Urbanismo, formó la Dirección de Obras Municipales de Ñuñoa en 1926, efectuando diversos trabajos en dicha Comuna, hasta 1927

Como Delegado del Gobierno de Chile al XIV Congreso Internacional de Planificación de Ciudades y de la Vivienda efectuado en Berlín el año 1931, tuvo una brillante participación. Aprovechó su estada en esta capital para trabajar en calidad de funcionario en el Servicio de Urbanización y Policía de Edificación durante seis meses. En esta ocasión preparó,entre otras conferencias, unensayosobre el problema de fundación de "NUEVAS POBLACIONESENTERRITORIODE COLONIZACION", en el cual se planteó el problema de los espacios vitales urbanos (Hinterland) en relación al proceso de desarrollo de la vialidad territorial en la empresa de una colonización. Este estudio despertó el más vivo interés en el Urbanista alemán Dr. Werner Hegerman, quien orientósu planteamiento hacia nuevos desarrollos. En estas reflexiones apreciamos el impacto de los trabajos de von Thünen y sus seguidores, quienes desde comienzos del siglo XIX destacaron importancia del estudio de la estructura del espacio rural en función de sus relaciones con el medio urbano.

Ocho años más tarde, con motivo del terremoto de Enero de 1939; Muñoz Maluschka publicó "CONCEPCION DINAMICA DEL URBANISMO: ESPACIOS VITALES URBANOS", donde la teoría que estudiaba fue aplicada en la zona devastada.

Cuando Brunner se incorporó el Departamento de Arquitectura de Obras Públicas en su Sección Urbanismo, dirigida por el Arquitecto José Luis Mosquera, -gran admirador de Otto Wagner-, Muñoz Maluischka trabajaba alli. Más adelante llegó a ser su Jefe, realizando una notable labor profesional. Desde este cargo asumió la contraparte de la Misión Brunner, acompañando al Profesor en los viajes de estudio realizados a diversas cludades del país y en sus conferencias universitarias y públicas en las cuales colaboró, además, como intérprete.

En 1936, Muñoz Maluschka viajó nuevamente a Alemania, donde profundizó sus estudios sobre las ciudades alemanas, cuyo crecimiento demográfico $\theta$ industrial no se había producido con caracteres de una explosión desordenada hacia el exterior, sino conforme aplanteamientosesquemáticos de expansión programada, en un contexto de eficiencia administrativa y conciliación de intereses públicos y privados.

De regreso a Chile, se empeñó en transformar la anacrónica Sección de Urbanismo del Ministerio de Obras Públicas en Dirección de Planeamiento. Esta, por su estructura, fué la Dirección de Planificación Territorial, cuyas facultades ampliaron tres veces el campo de acción del Urbanismo de la época. Intervino además, y de manera fundamental, en el proyecto de creación de la Corporación de la Vivienda, CORVI, concibléndola como el tercer eslabón ejecutivo de la acción planificadora, para la realización práctica de los Planos Reguladores.

Con motivo del terremoto de 1939, pasó a colaborar en la Corporación de Reconstrucción y Auxilio. En 1940, fué Director de la Asociación de Arquitectos de Chile, y en 1941. Secretario General del II Congreso Interamerlcano de Municipios. Particularmente obtuvo premios en diversos concursos para los que trabajó junto al Arquitecto René Aránguiz Saravia. Formó equipo de trabajo en diversas ocasiones con Alberto Schade y Rodulfo Oyarzún. Fué Urbanista Consultor de la Caja del Seguro Obrero. Es autor de "MANUAL DE URBANIZACION PARA ALCALDES Y DIRECTORES DE OBRAS MUNICIPALES", hablendo publicado numerosos estudios técnico-profesionales, y dictado conferencias en Europa, algunas de ellas también publicadas. Colaboró en diarios de Chile y del extranjero. Fué miembro del Instituto Nacional de Urbanismo, de la International Federation for Housing and Town Planning, con sede en Londres. Desde 1947 y hasta 1951 fué Profesor de la Universidad de Chile.

Desde Obras Públicas, llevó adelante importantes reformas urbanas y algunos proyectos de Brunner:

Remodelación de la Estación de Pirque, (Remodelación Parque Bustamante), que, de la Avda. Providencia se retira a la Avda. Matta, y se estructura tanto el Parque Bustamante de hoy, con la colaboración del Ingeniero J. Kelemen, como también la rotonda de la Plaza Baquedano y el arranque al oriente del Parque Japonés, con la colaboración del Paisajista Oscar Prager.

Apertura de la Diagonal Oriente en su tramo de Ñuñoa, con la intención de empalmar con Santa Isabel y estructurarla como vía alternativa del eje Providencia-Alameda.

Proyecto del Barrio Cívico de Santiago, a partir de algunas ideas de Brunner de despejar el Palacio de La Moneda, se procedio a expropiar la manzana completa que hoy constituye la Plaza de la Constitución y se establece además, por concurso públlco nacional, la fachada modulada y de altura uniforme.

Apertura de la Avda. Bulnes y renovación urbana entre la Alameda y Plaza Almagro mediante la Ley 8.412 de 1938, que introdujo la novedosa facultad de recuperar parte de plusvalia generada por el proyecto en el sector, el cual paso a completar el Barrio Civico iniciado al Norte de la Alameda.

Aplicación en otras comunas del Gran Santiago y del resto del país de las disposiciones de la Ley General de Construcción y Urbanización que obliga a las comunas de más de 7.000 habitantes a confeccionar, por cuenta de las Municipalidades, el Plano Regulador Urbano. Aquellas que disponian de recursos propios y personal técnico asi lo hicieron, como Providencia, Ñuñoa, Las Condes e incluso Santiago, que cumplio el requisito de Plano Oficial de Urbanización, traspasando el proyecto final de Brunner a planchetas catastrales escala 1:500. en 1939.

Expuso en su Cátedra de la Facultad, sus experiencias en materia de Planificación Urbana y Regional, donde destacan, además delo señalado, sus proyectos de acceso Norte a Santiago y el tramo inicial de la Circunvalación Exterior de esta cludad, incorporados en 1952-53 al plan intercomunal "Santiago una Gran Cludad", proyecto antes citado, de su discipulo Juan Parrochia. 
Entre 1946 y 1952, la Sección Urbanismo de la Dirección de Obras Públicas, a cargo de Muñoz Maluschkà , puso en aplicación más de 140 Planos Reguladores, contando con un equipo de cinco profesionales.

En 1952 Luis Muñoz viajó nuevamente a Alemania Occidental a profundizar sus estudios en Planificación Territorial. La experiencia planificadora recogida en sus visitas a este país, como huésped del Servicio Planificador, que había reconstruido el país demolido por la guerra a base de una Planificación Territorial científica y eficaz, le permitió hacer una labor precursora en la materia en Chile. Junto con la docencia impartida en la Universidad de Chile; Muñoz Maluschka participaba simultáneamente como profesor en estudiosy trabajos de Planificación Territorial con los planificadores del Estado alemán de Hannover. Refiriéndose a esta disciplina, señalaba a comienzos de los años cincuenta:

"La técnica de la Planificación Territorial es un resorte de la Administración Pública de un país para lograr el progreso general a través del uso óptimo del suelo y subsuelo territorial, científicamente establecido, y el emplazamiento más adecuado para el uso de las instalaciones técnicas, económicas y sociales de cualquiera naturaleza. Los regímenes democráticos alemanes son los que han desarrollado más en el mundo esta nueva técnica de una eficiente administración, que lleva los métodos usados hasta ahora para el Urbanismo a todo un territorio nacional y que, aplicado a la reconstrucción alemana de los años de la guerra, ha demos-

rrado su eticacıa practica".

Inspirado principalmente en sus estudios realizados en Baja Sajonia, realizó en el Instituto de Urbanismo de Chile, junto a otros destacados profesionales, los estudios de los aspectos urbanísticos, para la modificación de la Ley que dió a origen al D.F.L. 224, 1953, incorporándose por primera vez los conceptos y estructuras de la PLANIFICACION REGIONAL, MICROREGIONAL e INTERCOMUNAL, en Chile.

Esta fué, probablemente, su última gran obra, dado que el Gobierno del Presidente lbáñez, que él apoyaba, lo desplazó de su cargo por incomprensibles razones. Aún cuando había sido el principal promotor de las nuevas ideas de Planificación Territorial en Chile, quedó bruscamente sin su Jefatura en Urbanismo de Obras Públicas, sin su Cátedra de Urbanismo en la Universidad de Chile, y en consecuencia, sin respaldo para seguir su intercambio urbanístico con Hannover, en Alemania. Suúltima obra editada, y cuyo coautor es Carlos Trupp W., es PLANIFICACION INTEGRAL Y PLANIFICACION FISICA ENFRENTADAS ALA REALIDAD DE LA CONVIVENCIA HUMANA. Enella se desarrolla su doctrina genético-dinámica de Planificación, parte de la obra inédita \|lamada TRILOGIA DEL PAISAJE NATURAL.

Luis Muñoz Maluschka a quien Karl Brunner destacó y citó en su libro "Manual de Urbanismo", como uno de sus dos más valiosos colaboradores en Chile, y cuyos aportes en materia de Planificación Territorial fueron fundamentales en su época, murió en Madrid a comienzos de los años setenta, sin haber recibido el reconocimiento que su obra merecía.

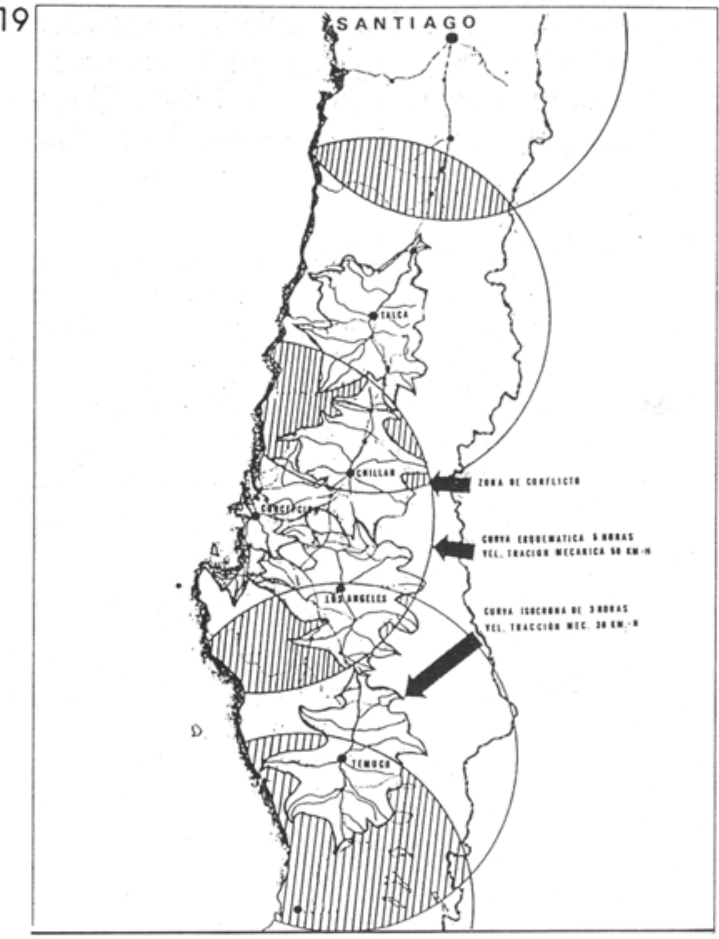

Los Arquitectos precursores, cuya labor aquí se ha evocado, fueron contemporáneos, aunque por sus edades, representaron generaciones diferentes. Actuando al mismo tiempo y sobre los mismos temas dieron enfoques complementarios a su acción. Nos parece destacable que sus vidas se empalmaron en vertical y en horizontal: ellos se sucedieron sin vacios en el tiempo, y, en múltiples ocasiones. constituyeron equipos profesionales para el desarrollo de proyectos de Arquitectura y Urbanismo. La labor que realizaron, contribuyó, de manera fundamental, a crear espacios de participación a los académicos y profesionales chilenos en el campo del Urbanismo, los que fueron ocupados con éxito y sin interrupción, durante los veinte años que siguieron al período que aqui se ha observado.

19. ESTUDIO DE CURVAS ISOCRONAS

20. EDIFICIO DE LA CAJA DE CREDITO AGRARIO EN EL BARRIO CIVICO, obra de los Aratos. LUIS MUÑOZM. Y RENE ARANGUIZ. (Foto de R. Chandia, 1991, U. Fotografia, Archivo y Medios Audiovisuales de la U. de Chile).

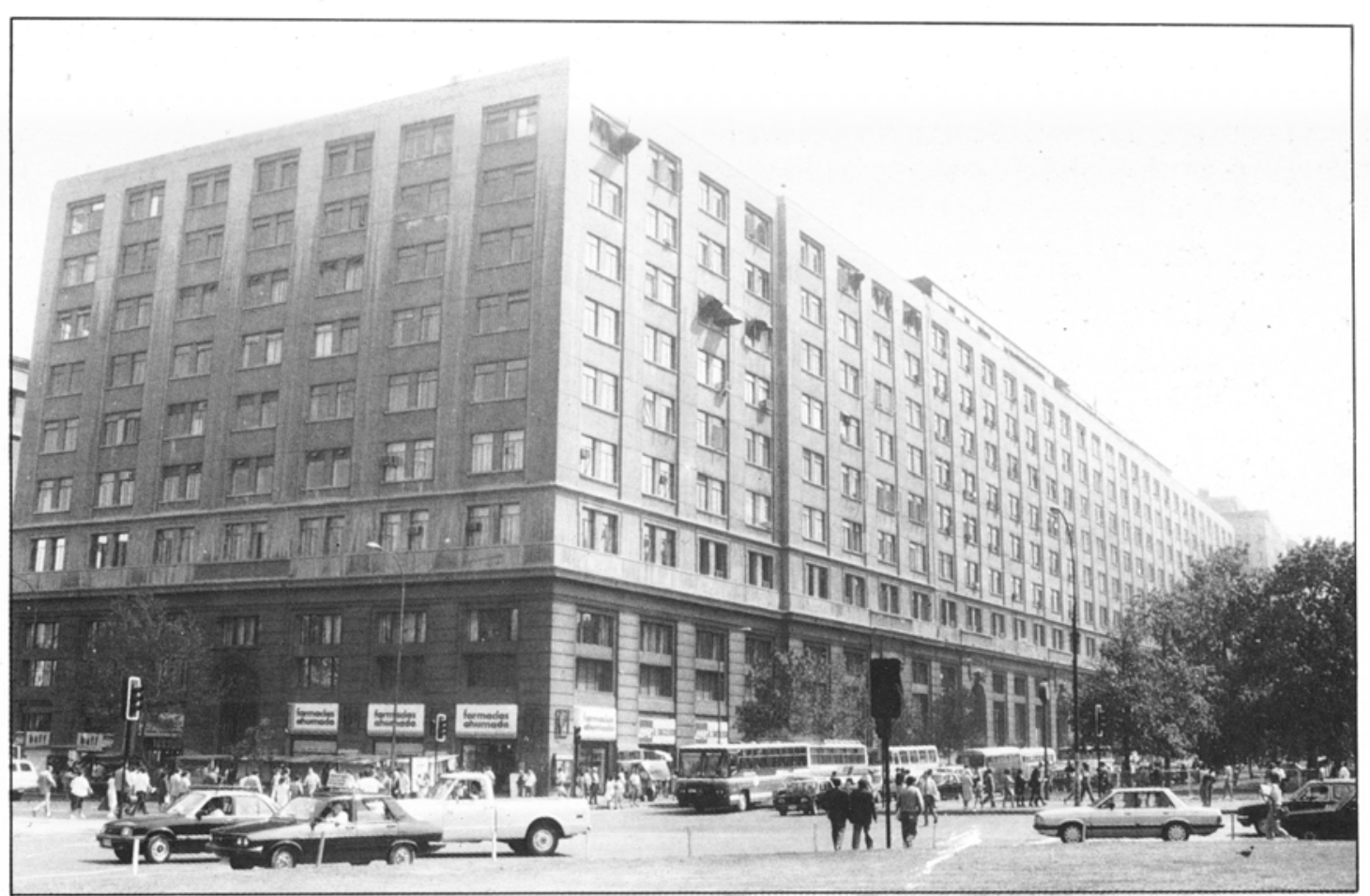

FUENTES DE INFORMACION CONSULTADAS:

BEDRAK, Moisés: "ElUrbanismo en Chile 1928 - 1973" en: REVISTA CA, N" 46 Stgo. Diciembre 1986

BRUNNER, Karl H.: "Santiago de Chile su Estado Actual y Futura Formo-

BRUNNER, Karl H.: "Manual de Urbanismo". Primer Tomo. Sintesis Las Viviendas Urbanas-Saneamiento, Ed. Concejo Municipal de Bogotó, Bogotó, 1939.

COLLINS, George R:; CRASEMANn COuUns, Christiane, "Camillo Sitte and Birth of Modern City Planning". Columbia University Studies in Art 法 de 1930, 1940. 1950

GUROVICH W. Alberto: "Antología del Urbanismo en Chile". DOCH mentos de Irabajo inéditos, Departamento de Urbanismo de la F.A.U. de la U. de Chile.

MUNZAGA V.. Gustavo: "Cronologíasobre Urbanismo y Diseno Urbano en Chile", P. Universidad Cotólica de Chile. Escuela de Arquitectura Separata Revista, EURE-Vol VI-Agosto 1980-N² 18. Ed. CIDU-IPU. MUNNOZ MALUSCHKA, Luis: "La transformacion de las Ciudades ChileNISMO, Edición Extroordinaria, de Diciembre de 1937.

MUNOZZ MALUSCHKA, Luis: "Concepción Dinómica del Urbanismo:

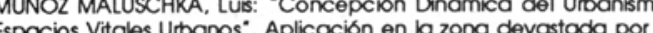
En: REVISTA URBANISMOY ARQ TECTURA, Asocioción de Arquitectos de Chile, № 10, Stgo, 1940. MUNNOZ MALUSCHKA, Luis: "Significado de la Planificación Territorial". Prefocio del Profesor, en: SEMINARRO de Investigación "Areas Veides en la Planificación", de Juan Parrochia Beguin F.A.U.., Universidad de Chile. Stgo, 1952

MUNNOZ MALUSCCHKA, Luis: TRUPP W., Carlos, "Planificocion Integral y Planificación Fisica Enfrentada a la Realidad de la Convivencia de obra no citada, y parte de la obra Inédita TRILOGLA DEL PAISAJE

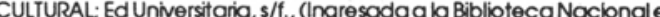
1983).

OYARZUN, Rodulfo.: "El Prof. Dr. Karl Brunner. su estada en Chile entre los años 1929-1934", en: REVISTA DE PLANIFICACION: VIVIENDA CIU DAD, REGION, № 7. IVUPLAN, F.A.U. Universidod de Chile, Stgo.. 1970 PARROCHIA B.. Juan: Los Origenes de la Planificación Intercomunal Micro-Regional y Regional en Chile. Documento de Trabajo inédito.

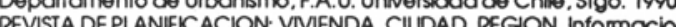
nes de diversos números ( $1^{2}$ al $8^{2}$ ). NUPLAN. F.A.U. Universidad de Chile, Stgo década de 1960 y año 1970

REVISTA URBANISMO Y ARQUITECTURA, Asociocion de Arquitectos de Chile, informaciones entregados en diversos números de 1930 y 1940. SCHADE, Alberto: 'Arquitectura Industrial', Escuela de Ingeniería, Unversidad de Chile, Ed. Galcon, 1954, tercera edicion.

sCHWEITZER, Angela, "Palabros de la Arquitecta Angela Schweitzer. en representación de los nuevos miembros vitalicios del Colegio de Arquitectos A.G., en: BOLEIINC.A. Septiembre, Octubre, Stgo... 1987.

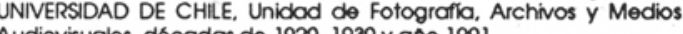

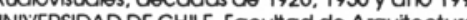
Urbanismo. Archtvos Fotorbficos CHLE, Focultod de Arqu

UNIVERSIDAD DE CHILE, Focultod de Arquitectura y Urbanismo. Archt. vos Generales del Departamento de Urbanismo (ex: IVUP. NUPLAN. DEPUR), ano 1949. y décadas de 1950, 1960 y 1970 Diversos documentos, en especial: Informes de Arqto. René URBINA VERDUGO, Dirección deIVUPLAN: "Reseñadel Desarrollo Institucional deIDEPUR", delArqto. ALFONSO RAPOSO MOYANO, e Informes del Arqto. Luis MUNNOZ MAUUSCHKA

UNIVERSIDAD DEL BIO-BIO, Focultad de Arquitectura y Construccion: "Rodulfo Oyarún PH. Arquitecto". Concepción. Abril de 1988. 